\title{
Source Authentication in Group Communication Systems
}

\author{
Xin Zhao \\ University of Michigan \\ 1301 Beal Ave, Ann Arbor, MI, 48105, USA \\ zhaoxin@eecs.umich.edu
}

\author{
Atul Prakash \\ University of Michigan \\ 1301 Beal Ave, Ann Arbor, MI, 48105, USA \\ aprakash@umich.edu
}

\begin{abstract}
Many group communication systems need to enforce a restriction that limits members are authorized to send messages to the group. Receivers therefore need to authenticate message sources before the received messages are accepted. Source authentication in peer-to-peer systems is trivial: the two communication parties can agree on one pair key and use this key to authenticate each other. However, because the group key is shared by all members in a group system, it is quite challenging to identify the sender and determine its authorization. Furthermore, if the authorization can be changed at run-time, source authentication problem can be even harder. This paper presents a source authentication technique called TTA scheme(Transitive Trust Authentication). TTA supports source authentication as well as dynamic authorization change. In addition, its computation and communication overhead is low.
\end{abstract}

\section{Introduction}

Many secure group communication systems only allow authorized members to send messages to the group. Therefore, a member must check the identity as well as the message source before accepting a received message. The source authentication process is trivial in a peer-to-peer communication system: the two communication parties can agree on one pair key and use it to authenticate each other. In a group system, the group key is shared by all members, which makes it challenging to identify the message source and determine its authorization. Furthermore, If the authorizations can be changed at run-time, the source authentication problem will be even harder.

Two most challenging questions are raised here:

1. How to generate unforgeable validity proofs? Because the group key is shared by all group members, symmetric encryption methods cannot generate unforgeable validity proofs. As an alternative, the digi- tal signature technique could guarantee the source authenticity. However, its computational overhead is prohibitively high. How to generate unforgeable validity proofs is therefore quite challenging.

2. How to support dynamic authorization change It is desirable to allow dynamic authorization change. For example, a member's sending right can be revoked at run-time. However, the receiver can authenticate the message correctly only if they have the updated authorization. In a large system, it is diffcult and expensive to distribute the policy changes to all group members in a reliable and secure way.

\section{Existing Solution}

Several reasonable solutions have been proposed to support single source authentication in group systems.

Canetti et al. proposed a $k$-MAC authentication scheme[1] which let the sender use $k$ MAC keys to compuate $k$ MACs for each message. Each receiver hold a subset of the $k$ MAC keys to verify the received messages. One major problem of this scheme is its computation and communication overhead since the sender needs to compute and send multiple MACs.

Gennaro and Rohatgi proposed a MAC Chain technique[2] to embed the MAC of a packet into its previous packet. If the initial packet is authenticated, the subsequent packets can be verifed at low cost. Its main shortcomings are its weak robustness to packet loss and lack of dynamic authorization change support. TESLA [3] [4], proposed by Adrian Perrig et al., is quite effcient. However, it requires that each receiver loosely synchronize with the sender when it joins the group. For a large group, the time synchronization may incur substantial delay. Moreover, TESLA does not support dynamic authorization change.

This paper presents the TTA(Transitive Trust Authentication) mechanism. It supports source authentication 


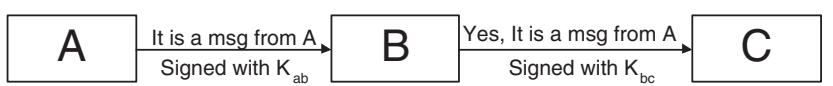

Figure 1. Transitive Trust Relation

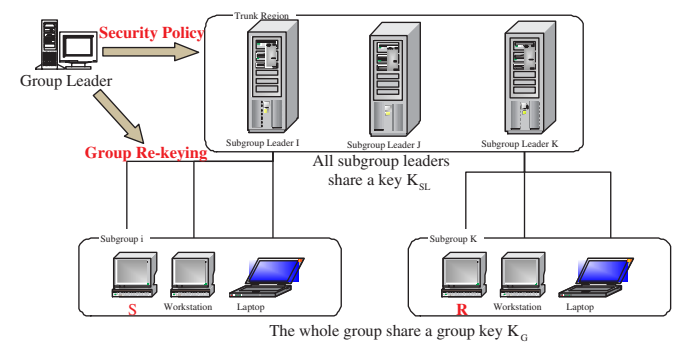

Figure 2. Infrastructure of TTA mechanism

as well as dynamic authorization change. In addition, we will show that the computation and communication overhead of TTA is low.

The rest of the paper is organized as follows: Section 3 gives the sketch of the TTA scheme, Section 4 describes one critical technique used in TTA. The performance analysis is put in Section 5. Finally, we reach the conclusion at Section 6.

\section{Transitive Trust Authentication Scheme}

\subsection{Transitive Trust Relation}

The basic idea of TTA is is illustrated in Figure 1: suppose a member $A$ wants to send a message to $C$, but $C$ accepts this message only if it believes that $A$ is a valid sender. However, $C$ might have no idea of $A$ 's authorization. To help $C$ authentication $A$, a mutually trusted member $B$ can be sets up to authenticate $A$ and certify the validity of $A$ to $C$. Because $C$ trusts $B$, it then trust $A$. This kind of trust relatioin is called textittransitive trust relation. The trust transition process is illustrated in Figure 1.

TTA extends the basic idea and organizes a multicast system as shown in Figure 2, four roles exist in the TTA organized system.

1. Group Leader:A many-to-many multicast group has one group leader, the group leader takes care of group administrative operations such as group re-keying, group policy changing, etc.;

2. Subgroup Leader: As Figure 2 shows, the group is divided into multiple subgroups. Each subgroup has one subgroup leader. The subgroup leaders trust one another. They authenticate and notarize the group messages sent in/out their subgroups. One shared key $K_{S L}$

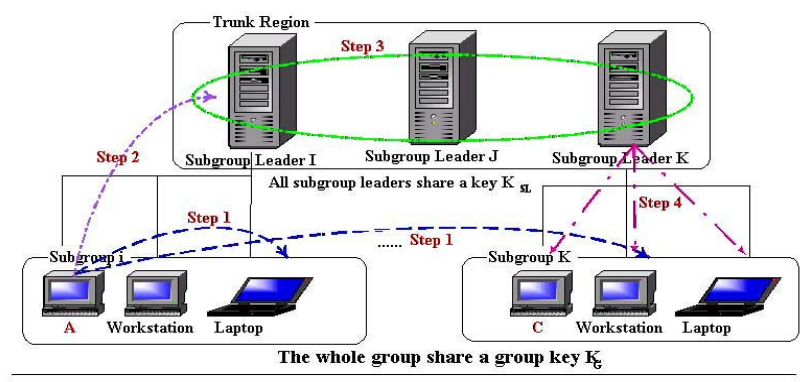

Step 1: $S \longrightarrow$ ALL GROUP MEMBERS

$\left\{U I D(S)\left|S N O_{S}\right| V_{K_{G}} \mid\{M\}_{K_{G}}\right\}$

Step 2: $S \longrightarrow S L_{S}$

$\underline{\left\{U I D(S)\left|S N O_{S}\right| V_{K_{G}}\left|H_{M}\right| H M A C_{K_{S, S L}}\left(U I D(S), S N O_{S}, V_{K_{G}}, H_{M}\right)\right\}}$

$\left\{U I D(S)\left|S N O_{S}\right| V_{K_{G}}\left|H_{M}\right| H M A C_{K_{S L}}\left(U I D(S), S N O_{S}, V_{K_{G}}, H_{M}\right)\right\}$

Step 4: Other subgroup leaders $\longrightarrow$ their children

$\underline{\left\{U I D(S)\left|S N O_{S}\right| V_{K_{G}}\left|H_{M}\right| H M A C_{K_{S L_{G}}^{i}}\left(U I D(S), S N O_{S}, V_{K_{G}}, H_{M}\right)|i| K_{S L_{G}}^{i-d}\right\}}$

Figure 3. Source Authentication Procedure in Many-to-Many Scenarios

is used by all subgroup leaders to certify the validity of messages to other subgroup leaders. Note that the subgroup leaders are not regarded as group members, they do not have the group key $K_{G}$, therefore, they cannot access the message content.

3. Group Member: Group members include senders and receivers. Each group member must be a receiver, but only members who are authorized to send messages are senders. Each group member belongs to one and only one subgroup, it only accept messages notarized by it own subgroup leader. Each sender $S$ share one paired key $K_{S, S L}$ with its subgroup leader $S L_{S}$.

The TTA system has the following trust relations:

- All group components, including senders, receivers and subgroup leaders, trust the group leader.

- The subgroup leaders trust one another.

- All group members trust their own subgroup leaders.

\subsection{Sketch of TTA scheme}

Figure 3 illustrates how TTA supports source authentication in many-to-many scenarios:

1. Step 1: The sender $S \longrightarrow$ other group members:

$$
\left\{U I D(S)\left|S N O_{S}\right| V_{K_{G}} \mid\{M\}_{K_{G}}\right\}
$$

The sender $S$ sends the message content packet to all group members. This packet includes: user ID 
$U I D(S)$, sequence number $S N O_{S}$, group key version number $V_{K_{G}}$ and the encrypted message content $\{M\}_{K_{G}}$.

Upon receiving this packet, the receivers buffer this message and wait for the corresponding message certifcate. To avoid unlimited waiting, a timer will be set for each buffered message, the valid message certifcate must arrive before the timer expires, or else the buffered message will be dropped.

2. Step 2: The sender $S \longrightarrow$ its subgroup leader $S L_{S}$ :

$$
\begin{gathered}
\left\{\underline{C} \mid H M A C_{K_{S, S L}}(\underline{C})\right\} \\
\underline{C}=\left\{U I D(S)\left|S N O_{S}\right| V_{K_{G}} \mid H_{M}\right\}
\end{gathered}
$$

$S$ frst generates the Message Certifcate $\underline{C}$ of this message. The message certifcate uniquely maps to the corresponding message. If the source of a message certifcate is valid, the source of the corresponding group message must be valid. Receivers then use the hashed value $H_{M}$ to verify the integrity of message content. After generating the message certifcate, it uses the paired key $K_{S, S L}$ to generate $H M A C_{K_{S, S L}}(\underline{C})$ which can be used by its subgroup leader to identify $S$.

The message certifcate as well as its HMAC is sent to $S$ 's subgroup leader $S L_{S}$. $S L_{S}$ authenticates the validity of $S$ through two steps: frst, $S L_{S}$ checks $S$ 's authorization record to see whether it has appropriate sending right. second, $S L_{S}$ identify the sender $S$ by checking the attached HMAC value. Through these two checks, $S L_{S}$ not only identifes the data source, but also enforces dynamic authorization control.

3. Step 3: The subgroup leader $S L_{S} \longrightarrow$ other subgroup leaders $S L_{G}$ :

$$
\left\{\underline{C} \mid H M A C_{K_{S L}}(\underline{C})\right\}
$$

If the message certifcate is verifed to be valid, the subgroup leader $S L_{S}$ will certify the validity of the message certifcate to all other subgroup leaders. $S L_{S}$ replace the HMAC value $H M A C_{K_{S, S L}}(\underline{C})$ with a new HMAC $H M A C_{K_{S L}}(\underline{C})$, which is computed with the $K_{S L}$ that is known by all subgroup leaders only.

The certifcate as well as the new HMAC will be distributed to all other subgroup leaders. Other subgroup leaders can check the HMAC to see whether the certifcate is notarized by a subgroup leader. Because all subgroup leaders trust one another, if HMAC is verifed to be generated by one subgroup leader, the corresponding message certifcate will be regarded as valid by all other subgroup leaders.

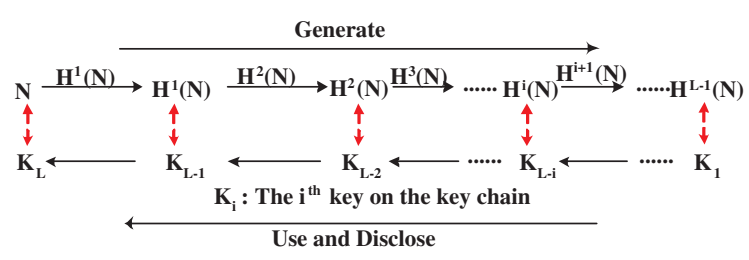

\section{Figure 4. Self-Authenticated One Way Key Chain}

4. Step 4: Other subgroup leaders $\longrightarrow$ their children:

$$
\left\{\underline{C}\left|H M A C_{K_{S L_{G}}^{i}}(\underline{C})\right| i \mid K_{S L_{G}}^{i-d}\right\}
$$

If the certifcate is verifed to valid, other subgroup leaders will certify the validity of the message certifcate to their children. Upon receiving the notarized certifcate, the receivers frst check whether this notarized certifcate came from their subgroup leaders. How can receivers authenticate whether the certifcate originated from their subgroup leader is actually a oneto-many source authentication problem and will be discussed in next section.

If the notarized message certifcate is verifed to be from its subgroup leader, receivers will be confdent that the source of the certifcate is valid. The source of the corresponding message will then be regarded as valid. The receivers can then use the hash value $H_{M}$, which is included in the message certifcate, to verify the integrity of message content. That £nalizes the whole message verifcation procedure.

\section{Source Authentication between Receivers and their Subgroup Leader}

TTA uses TESLA $[3,4,5]$ protocol to achieve source authentication between Receivers and their subgroup leader. It requires that each receiver synchronize with its subgroup leader. After the synchronization, the receivers can use their local time infer the upper bound of the subgroup leader's local time.

Bootstrap Stage As illustrated in Figure 4 shows, at the subgroup leader's bootstrap stage, the subgroup leader uses the self authenticated one-way key chain to generate a one-way key chain: the subgroup leader randomly pick a number $\mathrm{N}$ and repeatedly applying a one-way hash function $\mathrm{H}$ for $\ell-1$ times. It then gets a hash value chain: $H^{0}(N), H^{1}(N), \ldots H^{\ell-1}(N)$., where $H^{i}(N)=$ $H(H \ldots(H(N)$. The self-authenticated key chain is generated by putting the one-way hashed chain in reverse order. If we use $K_{i}$ to represent the $i^{t h}$ key on the key chain, 


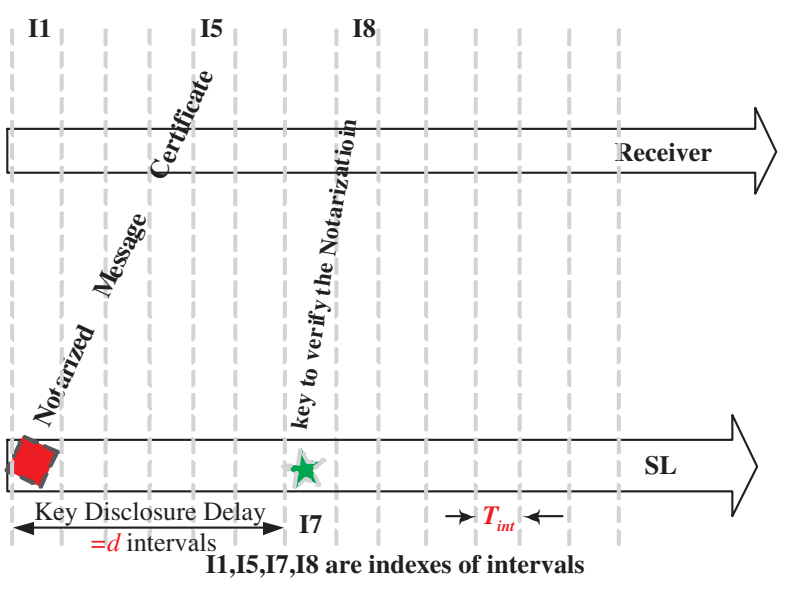

Figure 5. Source Authentication between a receiver and its subgroup leader

then $K_{i}=H^{\ell-i}(N)$. If $K_{i}$ is verifed to be valid, the validity of the key $K_{l}$ can be verifed by check whether $K_{l}=H^{i-l}\left(K_{i}\right) \quad l<i$. Therefore, the cost of key authentication is very low.

The subgroup leader's side As Figure 5 shows, the subgroup leader $S L$ splits time into even intervals, each interval is $T_{\text {int }}$ long. Interval 0 starts at time $T_{0}$, interval $i$ starts at $T_{i}=T_{0}+i * T_{i n t}$. At any time point, the subgroup leader should be in one interval $i$. Next,the subgroup leader has a self-authenticated one-way key chain $K_{S L}^{0}, K_{S L}^{1}, \ldots K_{S L}^{\ell-1}$. Each key $K_{S L}^{i}$ will be used to compute HMACs for message certifcates only within time interval $i$. Each used key will be kept secret for several(for example, $d$ ) intervals. After $d$ intervals, $S L$ discloses the key. The validity of the disclosed key can be verifed with the self-authenticated key chain technique.

The receiver's side $\underline{C}$ represent the message certifcate $\left\{U I D(S)\left|S N O_{S}\right| V_{K_{G}} \mid H_{M}\right\}$. When the receiver receives notarized certifcate $N_{j}$ sent in interval $i$ at local time $t_{r}$, the packet $N_{j}$ should be like this:

$$
\left\{\underline{C} \mid \underline{H M A C_{K_{S L}^{i}}(C)|i| K_{S L}^{i-d}}\right\}
$$

The receiver takes the following steps to check the notarized certifcate arrived safely:

1. Infer the time interval $i$ the subgroup leader was in when it sent out the notarized certifcate. Since $i$ is included in the packet, the receiver can verify the $i$ by checking the authenticity of the disclosed key $K_{S L}^{i-d}$. If the last authenticated key is $K_{S L}^{l}$, the authenticity of $K_{S L}^{i-d}$ can be determined through checking whether $K_{S L}^{l}=H^{i-d-l}\left(K_{S L}^{i-d}\right)$.
2. Compute the upper bound of the sender's current time interval $x$. Because the receiver is loosely time synchronized with the sender, the receiver can compute the upper bound on the sender's clock $t_{s}=t_{r}+\Delta$, and consequently calculate the maximal interval with $x=\left\lfloor\left(t_{s}-T_{0}\right) / T_{\text {int }}\right\rfloor$.

3. Compare $i+d$ and $x$, it $x<i+d$, this key used to notarize this message certifcate must be still a secret and the notarized certifcate arrived safely. Otherwise, probably the key have been disclosed, and the certifcate notarization could be forged with the revealed key.

If the notarized certifcate is assured to arrive safely, the receivers will buffer it and wait for disclosure of the key $K_{S L}^{i}$ to authenticate the packet source.

After the key $K_{S L}^{i}$ is disclosed, the receiver frst check the validity of $K_{S L}^{i}$. If the key $K_{S L}^{i}$ is valid, it can be used to check the message certifcate's HMAC $H M A C_{K_{S L}^{i}}(C)$. If it is valid, the source of the message certifcate is valid, and the source of the corresponding message is also valid. We follow TELSA's suggestion to set $d$ as $\left\lceil R T T / T_{\text {int }}\right\rceil+1$, here, $R T T$ is a reasonable upper bound on the round trip time between the receiver and the subgroup leader.

\section{Performance analysis}

Communication Overhead If TTA scheme uses 80 bit HMAC-MD5 to generate HMACs, both the disclosed key and hashed value are 10 bytes long. The user ID, sequence number, key version number, and interval index are 4 bytes long. The sender's subgroup leader needs to send/receive message certifcates for 3 times. The communication cost is 128 bytes/message. Other subgroup leaders only need to send/receive the certifcate twice. The communication cost is $90 \mathrm{bytes} / \mathrm{message}$.

Computation Overhead We also use simuation program to evaluate TTA's computation overhead. The simulation program is written in $\mathrm{C}++$ and runs on a $1.4 \mathrm{GHz}$ Pentium IV Linux PC, cryptography library is OpenSSL[6]. The simulation results show that the experimental machine can process 61900 messages per second. If the average length of messages is 1000 bytes, The experimental machine can process the group messages of around $61.9 \mathrm{M}$ (Bytes/second).

The generation of key chain is effcient too. If the interval length is 0.1 seconds, computing a key chain long enough to notarize certifcate for one hour only takes the subgroup leader around 0.12 (seconds).

Verifcation Latency and Probability Verifcation latency is defned as follows: when a group member receives a group message, it records this time as the starting point. 


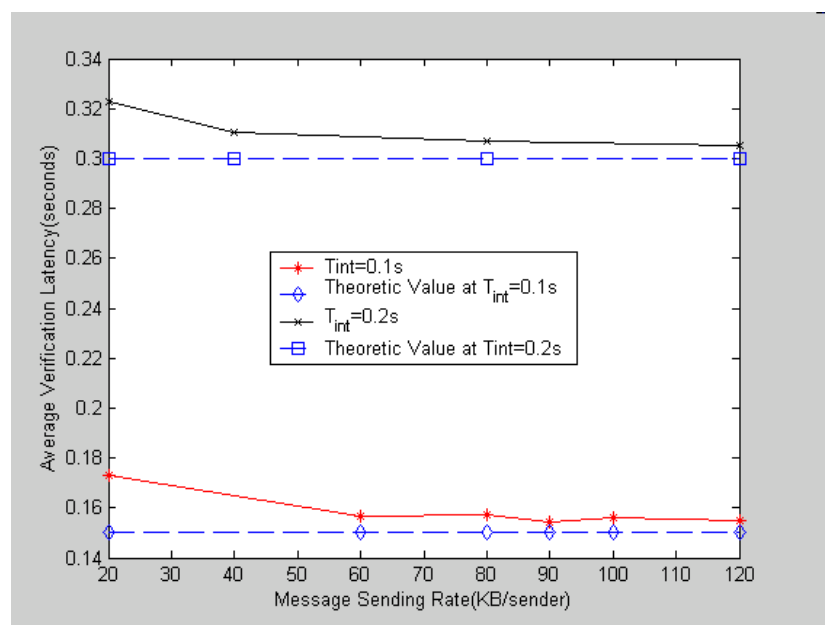

Figure 6. Average Authentication Latency

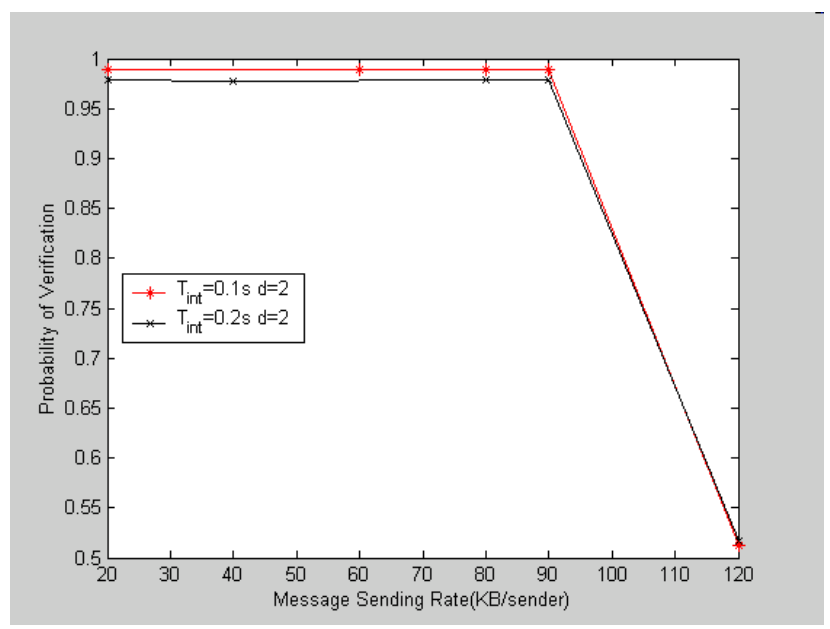

Figure 7. Veri£cation Probability

The time that the message source is verifed is recorded as the ending point. The verifcation latency is the time elapsed between the starting point and ending point. The dominate factor of verifcation latency is the notarization key disclosure delay. Assuming that the notarized message certifcates arrive in constant rate. Under such condition, the average key disclosure latency should be equal to $T_{i n t} *(d-0.5)$.

The simulation program is built up on the NS2(Network Simulator 2) [7] platform, the hardware is a $1.4 \mathrm{GHz}$ Pentium IV Linux PC. In the simulation environment, 200 members are divided evenly into two subgroups. 20 out of these 200 members are senders. 10 senders for each group. Each member has a $5 \mathrm{MB}$ connection with its subgroup leader, and leaders maintain an $1 \mathrm{MB}$ connection with each other. The network transmission latency is $3 \mathrm{~ms}$.

The simulation result shown in £gure 6 con£rms our theoretic analysis. Moreover, we can that different message sending rates has little effects on average verifcation latencies. The dominant factor is still the key disclosure delay.

The verifcation probability is the ratio of the number of messages verifed to the number of messages sent. Because the successful verifcations of TTA scheme rely on the delivery of both the message and its certifcate, when network is congested, the verifcation failure rate will be doubled. We increase the message sending rate to saturate the network and expect to see that TTA's verifcation probability will drop fast when network is congested. Figure 7 confrmed that the verifcation probability is very high when network bandwidth is still available. However, when the traffc saturates the network, the verifcation probability drops fast. How to make the TTA system more fault tolerable will be our next research topic.

\section{Conclusion}

TTA converts the procedure of many-to-many source authentication into two peer-to-peer source authentication procedures and a one-to-many source authentication procedure. With this conversion, TTA can offer source authentication, dynamic security policy enforcement, and minimal overhead.

\section{References}

[1] Ran Canetti, Juan Garay, Gene Itkis, Daniele Micciancio, Moni Naor, and Benny Pinkas. Multicast security: A taxonomy and some effcient constructions. In $I N$ FOCOMM'99, 1999.

[2] Rosario Gennaro and Pankaj Rohatgi. How to sign digital streams. In Advances in Cryptology C CRYPTO 97, 1997.

[3] Adrian Perrig, Ran Canetti, J.D̃. Tygar, and Dawn Song. The tesla broadcast authentication protocol. RSA CryptoBytes, 5(Summer), 2002.

[4] Adrian Perrig, Ran Canetti, J. D. Tygar, and Dawn Xiaodong Song. Effcient authentication and signing of multicast streams over lossy channels. In IEEE Symposium on Security and Privacy, pages 56-73, 2000.

[5] A. Perrig, R. Canetti, D. Song, and J.D. Tygar. Ef£cient and secure source authentication for multicast. In Network and Distributed System Security Symposium, NDSS '01, February 2001.

[6] The OpenSSL Project. http://www.openssl.org.

[7] The Network Simulator - ns-2. http://www.isi.edu/nsnam/ns. 